2018-06-25

\title{
QualitySDN: Improving Video Quality using MPTCP and Segment Routing in SDN/NFV.
}

\section{Barakabitze, Alcardo Alex}

http://hdl.handle.net/10026.1/12538

\subsection{9/NETSOFT.2018.8459917}

NetSoft

IEEE

All content in PEARL is protected by copyright law. Author manuscripts are made available in accordance with publisher policies. Please cite only the published version using the details provided on the item record or document. In the absence of an open licence (e.g. Creative Commons), permissions for further reuse of content should be sought from the publisher or author. 


\title{
QualitySDN: Improving Video Quality using MPTCP and Segment Routing in SDN/NFV
}

\author{
Alcardo Alex Barakabitze, Is-Haka Mkwawa, Lingfen Sun and Emmanuel Ifeachor \\ School of Computing, Electronics and Mathematics, Plymouth University, UK \\ E-mail: \{alcardoalex.barakabitze, is-haka.mkwawa, L.Sun, E.Ifeachor\}@ plymouth.ac.uk
}

\begin{abstract}
In this paper, we present a novel QoE-aware SDN/NFV system by utilizing and integrating Multi-path TCP (MPTCP) and Segment Routing (SR) paradigms. We propose a QoE-based Multipath Source Routing (QoEMuSoRo) algorithm that achieve an optimized end-to-end QoE for the end-user by forwarding MPTCP subflows using SR over SDN/NFV. We implement and validate the proposed scheme through DASH experiments using Mininet and POX controller. To demonstrate the effectiveness of our proposal, we compare the performance of our QoE-aware MPTCP SDN/NFV SR-based proposal, the MPTCP and regular TCP in terms of system throughput and the end-user's QoE. Preliminary results shows that, our approach outperforms the other aforementioned methods.
\end{abstract}

Keywords-SDN, NFV, Quality of Experience (QoE), Segment Routing (SR), MultiPath TCP (MPTCP), Video Quality

\section{INTRODUCTION}

Multipath TCP (MPTCP) [1], [2] has emerged as the transport protocol capable of forwarding data traffic using multiple paths. MPTCP has recently been standardized by the IETF, and its implementation in the Linux kernel is already available [3]. The main idea of MPTCP is to strip data traffic to multiple disjointed paths inside the network, by creating multiple subflows for a single transport connection. The receiving side that supports MPTCP aggregates the transmitted sub-flows and reassembles the packets that originate from different paths. As shown in our previous work [2], MPTCP implementations can be integrated by new cuttingedge technologies such as SDN and NFV in order to achieve load balancing, security, reliable communication and better network resources utilization that leads to higher network throughput and the end-user's QoE [1], [4].

Despite the above mentioned benefits of MPTCP, the key technical aspects that affect the performance of this transport layer protocol is the lack of control and routing mechanisms of the splitted subflows. In that aspect, a centralized SDN controller looks to be a fundamental tenet for routing the MPTCP subflows. Intuitively, the SDN controller that maintains the global view can provide programmable environment to implement intelligent QoE-based routing mechanisms for the MPTCP subflows [5]. However, similar to how Border Gateway Protocol (BGP) tables have grown with the spread of the Internet, TE implementations in the data plane results into the number of rules on every switch to grow tremendously. It is worth mentioning that, SDN switches are incapable to handle large volume of flow rules because the complex rule matching 978-1-5386-4633-5/18/\$31.00 (C)2018 IEEE in SDN (e.g., wildcards) requires switches to store rules in TCAM, which is expensive, limited in size and needs high power consumption [6]. The limitation of SDN switches to store large number of rules can be greatly solved by Segment Routing (SR) [7] where a logical path of MPTCP subflows can be expressed as a sequence of segments between the ingress and egress network nodes (e.g., a switch/router/link). SR has been recently proposed by the IETF to provide TE by simplifying control plane where SDN switches no longer need to maintain per-demand routing information.

In this paper, we provide original practical TE solutions by using MPTCP and SR in SDN/NFV networks. We aim to facilitate efficient routing and speed up the transfer of large amount of multimedia applications between end-points. To improve the video quality, we propose to use multiple shortest paths for MPTCP subflows transmission and apply the Multi-flow commodity and Constrained Shortest Path Model (MCSPM) to choose important intermediate nodes to perform source routing using SR paradigm. We extend our work in [2] where we demonstrated that utilizing MPTCP/SR in SDNbased networks can improve system performance for video streaming services. In this paper, we extend the concept to further consider the NFV implementation in our proposal. The contributions of this paper are 2-fold:

- We propose the QoE-aware MPTCP SDN/NFV SR-based system that provide an efficient orchestration, QoE control and management of future multimedia services.

- We present our system model and a QoE-based multipath source routing algorithm called "QoEMuSoRo" that forward traffic using SR paradigms over the proposed SDN/NFV system.

The rest of this paper is organized as follows: Section II provides related work while Section III presents the proposed QoE-aware MPTCP SDN/NFV SR-based system. Based on MCSPM and the concept from graph theory, we formulate our SDN/NFV system model and present the proposed QoE-based multipath source routing algorithm in section IV. Section V $\mathrm{V}$ presents the performance and evaluation of the proposed SDN/NFV system. Finally, section VI concludes the paper.

\section{RELATED WORK}

\section{A. MPTCP SDN/NFV-based Approaches}

An Openflow-based SDN controller is used in [5] to implement a MPTCP path manager where switches are configured to 
forward subflows over different paths. The SubFlow Optimizer (SFO) is presented in [8] to enable load balancing by providing MPTCP hosts an optimum number of subflows and assign the best paths to new established subflows in SDN environment. Using the Linux's Kernel-based Virtual Machine (KVM), the behavior and performance of a virtualized proxy as an instance of NFV is analyzed in MPTCP connections over cellular network [9]. A Multi-flow update problem is formulated as a mixed integer programming in [4] to reduce the update time of flows in Software Defined NFV (SDNFV) systems. We note that, none of these MPTCP-based SDN/NFV solutions consider to improve the end-user's QoE using real-time video communication. In addition, as mentioned earlier, MPTCP implementation in SDN/NFV would results into installing large number of flow rules in switches leading to overhead at the controller and increased deployment cost. We believe that SR is an appealing solution for reducing the number of forwarding rules taking the fact that each SDN switch on the path needs to have an entry for a traffic-demand to forward its packets to the next hop.

\section{B. Segment Routing SDN/NFV-based Approaches}

SR provides capabilities to reduce the number of forwarding rules by encoding Traffic Engineering (TE) paths into SR paths. SR in [6] is reported to save up to $88 \%$ forwarding rules overhead and handled more than $92 \%$ flow updates in real-time compared to traditional solutions. A recent SDN [10] SR-approach, indicate an approximately 50\% reduction in the number of control messages between source and destination nodes. Practical TE through shortest paths in SDN WANs using SR is employed in [11] where only few intermediate nodes are selected to route all traffic in the network. Motivated by the capabilities of SR to reduce flow rules, we introduce a QoE-aware MPTCP SDN/NFV SR-based system that provide an efficient orchestration, QoE control and management of future multimedia services.

\section{Proposed QoE-AwARE MPTCP SDN/NFV-BASED SR SYSTEM}

In order to meet the aspects of QoE control and management of multimedia services in future networks, we propose a novel QoE-aware MPTCP SDN/NFV-based SR system shown in Fig 1 that achieve an optimized End-to-End QoE level for the end-users. Our proposal can provide multiple disjointed shortest paths for MPTCP subflows and perform source routing using SR paradigm. More importantly, it can learn and adapt to changing network conditions or media contents. The proposed SDN/NFV system consists of the Data plane and the QoE-control and management plane.

\section{A. Data Plane}

The data plane consists of SDN switches that support SR technology. It forms the data acquisition layer which consists of a set of different Virtual Network Functions (VNFs) arranged together depending on a set of network traffic flows. It also represents the forwarding layer of the network

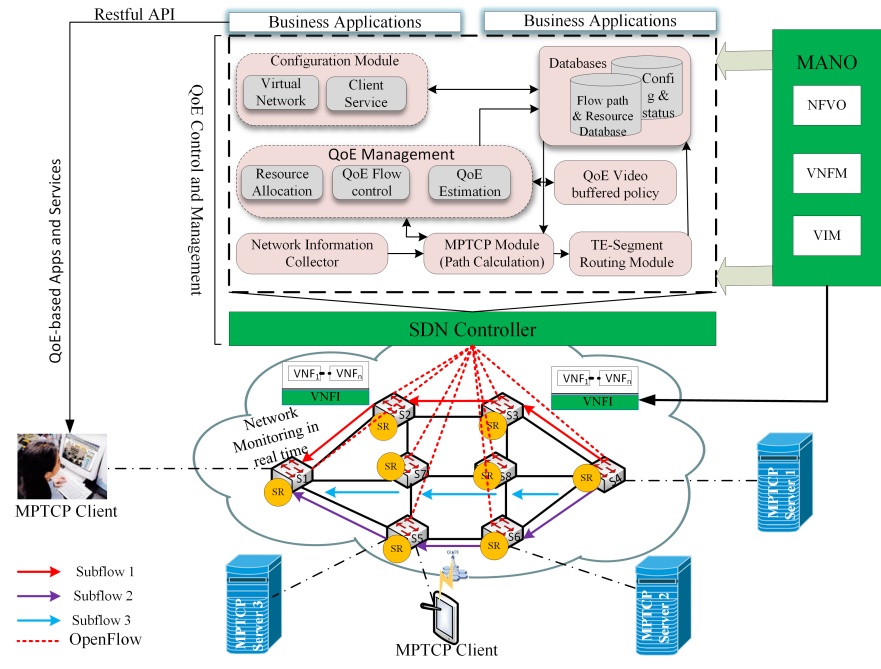

Fig. 1. The Proposed QoE-aware, MPTCP SDN/NFV-based SR System. MANO is the NFV management and orchestration framework consisting of three functional blocks: the NFV Orchestrator (NFVO), Virtual Infractructure Manager (VIM) and VNF Manager

where many software/hardware devices (e.g.,virtual routers and virtual switches) are interconnected using virtual or wired connections or common wireless radio channels.

\section{B. QoE Control and Management Plane (QoCoMa)}

We extended the POX controller with three functional modules, namely, the MPTCP-based flow manager module, the TE-Segment Routing module and the QoE management module.

1) Network Information Collector (NIC): This module performs collection of network information and QoE requirements of end-users, system events and any network topological changes (e.g., during link or node failure).

2) MPTCP Module: This module computes the shortest paths and then performs path allocations to MPTCP subflows. Based on the collected link information, the MPTCP module communicates with the $\mathrm{QoE}$ management module so that resources can be assigned to the calculated paths of subflows to meet their QoE requirements. Instead of installing these subflow paths in SDN switches as forwarding rules, we use SR approach where the forwarding table of the ingress switch is configured with an ordered list of segments [7]. The ingress switch then add labels with an ordered list of segments to a packet header and forwards it to its destination point.

3) TE-Segment Routing Module: To implement the TESR module that maps the computed subflow paths to SR paths, we consider the SR assignment algorithm presented in [7]. For example, given a multimedia flow $f$ requested by a MPTCP client whose ingress node is OpenFlow switch $S 1$ and the engress node is switch $S 4$, then the complete path for subflow $s f 1$ with intermediate nodes $\left\{S_{2}, . . S_{N-1}\right\}$ is: $P_{s f 1}=\{\mathrm{S} 4 \rightarrow \mathrm{S} 3 \rightarrow \mathrm{S} 2 \rightarrow \mathrm{S} 1\}$. With reference to Fig 1, the algorithm takes this path and the graph of the topology as inputs. It maps the specific path of the subflow and returns 
the segment list as an output of the assigned SR paths. More details of SR implementations are available in our previous work in [2].

4) QoE-Management Module: The QoE management performs various network management and ensures that the overall KPIs of the SDN/NFVs system are met. It also ensures that proper resource allocations, QoE-flow control and QoE estimation related to multimedia applications is achieved. In order to ensure the overall system performance of a multimedia service, the QoE management module takes measurements of KPQ/Is related to QoE metrics such as stalling, video quality and network metrics such as packet latency, jitter, network throughput and packet loss. It also performs autonomic fault detection and recovery mechanisms for ensuring that any point of failure in the SDN/NFV can be configured and recovered without affecting the end-user's QoE service delivery chain. The NFV management and orchestration (MANO) is responsible for managing and orchestrating virtualized network functions (VNFs).

5) Configuration Module: This module provides interfaces for virtual and physical network resources setup. It also provides interfaces for end-users service QoE configurations (e.g., QoS/QoE-based flow transmission rule for multimedia applications, throughput, packet loss). The configurations of these resources (for virtual and physical) and their associated QoS/QoE policies are stored in the configuration and resource databases (see Fig 1).

6) Database Module: The configuration parameters, monitoring status reports and different fine-grained resources are stored in the database module. It also maintains all SR subflow paths with their QoE requirements. When a new subflow of a MPTCP connection is uploaded to the SDN controller, the module queries in the database to look for an existing path that corresponds to this MPTCP connection. If the path exists, the subflow is allocated to a specific path of a previously assigned subflow's path of the same MPTCP connection, otherwise a new path computation for this subflow is performed using the MPTCP-flow manager/module. The new path is mapped to the SR paths and stored in the database so that it can be used later by subflows of the same MPTCP connection.

7) The SDN Controller: The SDN controller is implemented as an extension to POX controller. Computations of shortest paths of subflows is performed using the MPTCP module when the request is uploaded to the controller. The controller allocates the subflows to transmission paths using the MPTCP module and then maps these subflow paths to SR paths using the TE-SR module (see in Fig 1).

\section{SYSTEM MODEL AND QOE-BASED MULTIPATH SOURCE ROUTING ALGORITHM}

We consider an SDN/NFV network represented as a directed graph $G=(V, E)$ where $V$ is the set of nodes (switches or VNFs) and $E$ is the set of edges. Each $e \in \mathrm{E}$ is associated with a non-negative integer link weight denoted by $W(e)$. In real world network scenarios, the link weights indicate a non-fixed parameters such as link bandwidth, packet loss, delay or link utilization. We use such information to compute the optimal multiple set of $h$ shortest paths from source to destination. We denote $b_{w}$ as the available link bandwidth connecting a pair of SDN/NFV nodes and $B_{s f}^{k}$ as the required bandwidth of a subflow $s f$ of an MPTCP connection $k$. The shortest path of a traffic-flow demand $f$ from the source $s \in \mathrm{V}$ to destination $t \in \mathrm{V}$ is denoted by $p(s, t)$. We consider a Multi-flow commodity and Constrained Shortest Path Model (MCSPM) [12] that enables to find the optimal shortest routes for the multimedia flows from source to destination based on the defined specific constraints for a given service. The aim of MCSPM is to find a set of nodes with minimum link weight subject to multimedia flow constraints and perform better utilization of the network resources. As shown in [12], we also define the weight, $W e_{i j}$ of link $(i, j)$ in this paper as the sum of delay $\left(d l_{i j}\right)$ and packet loss $\left(p l_{i j}\right)$ values of a link multiplied with their scale factors $\alpha, \beta \geq 0$ respectively such that:

$$
W e_{i j}=\alpha \times d l_{i j}+\beta \times p l_{i j}, \forall(i, j) \in E
$$

While considering the MPTCP subflows of video service that have to be routed from MPTCP server to an MPTCP client, we define an optimization objective function that route all subflows in the network through shortest paths with minimized cost as defined in equation 1 . The objective function is defined as:

$$
O_{f}=\min \sum_{(i, j) \in E} \sum_{k \in K}\left(W e_{i j} s f_{i j}^{k}\right)
$$

Where $s f_{i j}^{k}$ indicates the subflow of a MPTCP connection $k$ routed on link $(i, j)$. We define the following parameters that are used to formulate the objective function constraints of which because of space are not shown in this paper.

We consider a multimedia flow $f$ consisting of subflows $s f_{1}$, $\left.s f_{2}, \ldots ., s f_{N}\right\}$. In order to route these subflows of an MPTCP connection, we first find the shortest paths between end-points in the network based on the link weight and bandwidth of the link as well as the QoE-demand of an MPTCP request. In real-world network operation scenario, a link may become a bottleneck when it can not provide the required resources or is likely to be the most used path for any pair of communicating nodes within an SDN/NFV networks. We introduce the link criticality $l_{c}$ parameter in order to balance the load in the SDN/NFV system and make sure that we avoid a bottleneck link. Suppose that $P_{s t}$ is the set of the first $h$ computed shortest paths from a MPTCP server to MPTCP client, and let also $P_{s t}(\mathrm{c})$ be the number of times that link $e \in \mathrm{E}$ is included in the first $h$ shortest paths. The occurrence rate of link $e$ in the first $h$ shortest paths can be computed as $P_{s t}(\mathrm{c}) / h$ for any pair of communicating nodes. The total expected load on link $e$ can be expressed as the sum of the expected number of trafficdemands on that link from all possible paths connecting the 
source and destination in an SDN/NFV system. This way, the link criticality $l_{c}$ can be defined using equation 3 .

$$
l_{c}=\sum_{(s, t) \in T D} P_{s t}(c) / h
$$

$T D$ is the traffic demand that is recorded and stored in the database in every time $T$. We use the link criticality which is updated by the controller after every time $T$ to balance the load in the network. When all the shortest paths have been computed based on the described link constraints, then the controller can map and assign these MPTCP subflows into SR paths. The MPTCP connection is then started where QoE metrics can be reported during video streaming. Algorithm 1 summarizes the overall steps of the proposed approach using QoE-aware MPTCP/SR over SDN/NFV network.

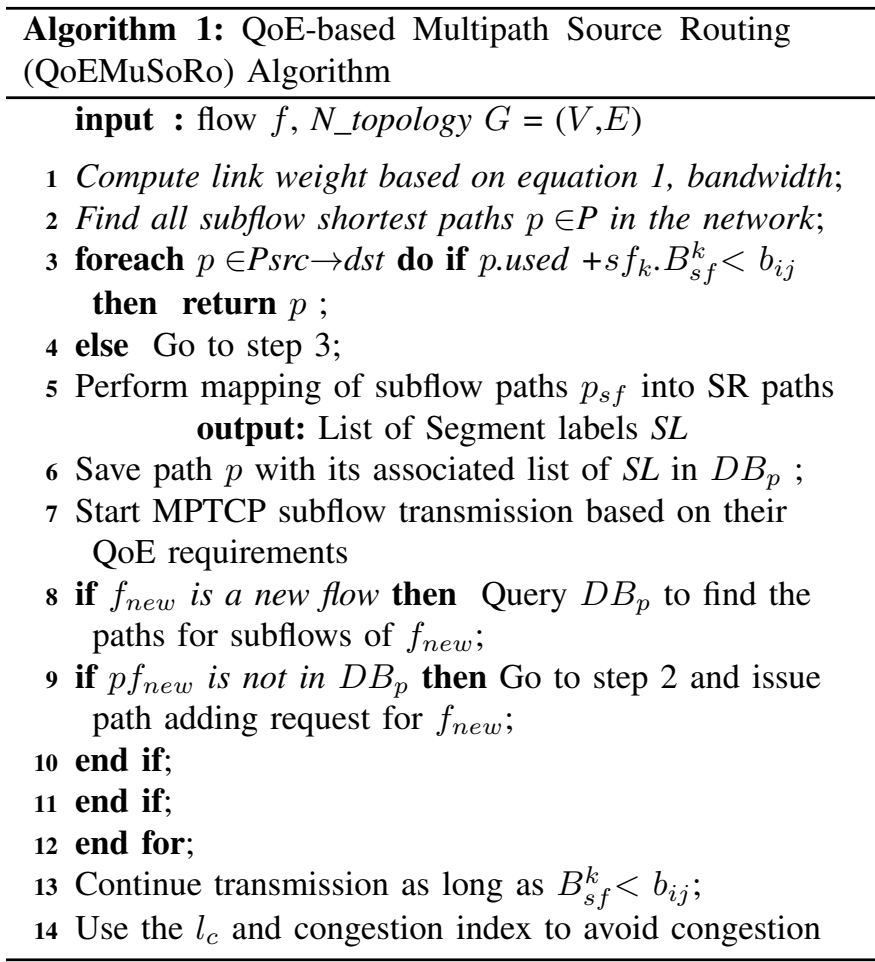

\section{PERformance And EVAluation}

\section{A. Experimental Testbed \& Setup}

We use an experimental testbed shown in Fig 2 which consists of the edge, aggregation and core layer consisting of 8,4 and 2 SDN switches respectively. Two VMs both running Linux (Ubuntu V16.04 LTS) were installed with the MPTCP v0.92. The mininet that used to model the network redundant

TABLE I

LIST OF USED PARAMETERS

\begin{tabular}{|l|l|l|l|}
\hline N.Layer & Bandwidth (Mbps) & Delay (ms) & Packet Loss (\%) \\
\hline Edge & 3 & 15 & 1 \\
\hline Aggregation & 2 & 20 & 3 \\
\hline Core & 3 & 30 & 3 \\
\hline
\end{tabular}

links at each level as shown in Fig 2 was installed in another VM. Redundant links are used in datacenter networks to enable the implementations of multipath routing in SDN/NFV systems. The POX controller was installed in the second VM. The SR module was implemented following a customization of source codes available at [13].

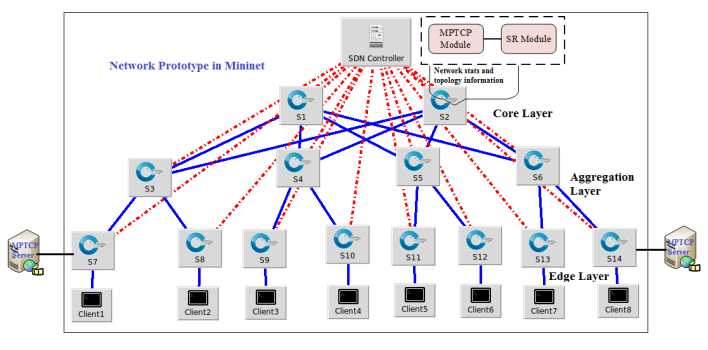

Fig. 2. Experimental Testbed.

The "Big Buck Bunny" video was encoded using ffmpeg version 3.3.4 with the libx265 at 3 different resolutions (720p, $480 \mathrm{p}$ and $360 \mathrm{p}$ ) with video encoding rate of $2.496 \mathrm{Mbps}$, $1.536 \mathrm{Mbps}$ and $1.0888 \mathrm{Mbps}$ respectively. We set the link parameters as shown in Table I. When the MPTCP is enabled, we keep the default configurations of MPTCP V0.92 and configure the MPTCP path manager to a full-mesh to limit each MPTCP connection to have only 3 MPTCP subflows. We use the VLC-DASH plugin [14] as the DASH client for collecting and reporting the performance of video quality and throughput during video streaming. The system throughput is computed from Payload_bits/download_time; where a Payload_bits is the number of extracted bits of the video content per single unit time. Apache Server was installed on two machines that supports MPTCP. These machines were then attached on mininet network as shown in Fig 2. The video transmission is repeated 40 times from MPTCP client to MPTCP server. We compare our proposal with the MPTCP and regular TCP in terms of throughput and the end-users-QoE.

\section{B. Experimental Results and Discussion}

1) System Throughput: Fig 3 shows the comparison of system throughput of the QoE-aware MPTCP SDN/NFV SRbased proposal, MPTCP and the regular TCP for video resolution of $360 p, 480 p$ and $720 p$. For each video transmission, the throughput of our proposed approach is higher compared to MPTCP and the regular TCP. While the performance of MPTCP exploits multipath transmission, the QoE-aware MPTCP SDN/NFV SR-based performs better because of employing SR which does not require any path signaling. Table II shows the average throughput achieved by QoE-aware SDN/NFV SR-based, MPTCP and TCP for 360p, 480p and $720 \mathrm{p}$ after 40 runs of video streaming.

2) Video Quality Measurements: For video quality, we use the reception quality $(\rho)$ [15] metric defined as the ratio between download throughput and video encoding rate. If $(\rho)$ $>1$ then the video has good reception quality otherwise, the video has poor quality. Fig 4 shows the comparison of a video 

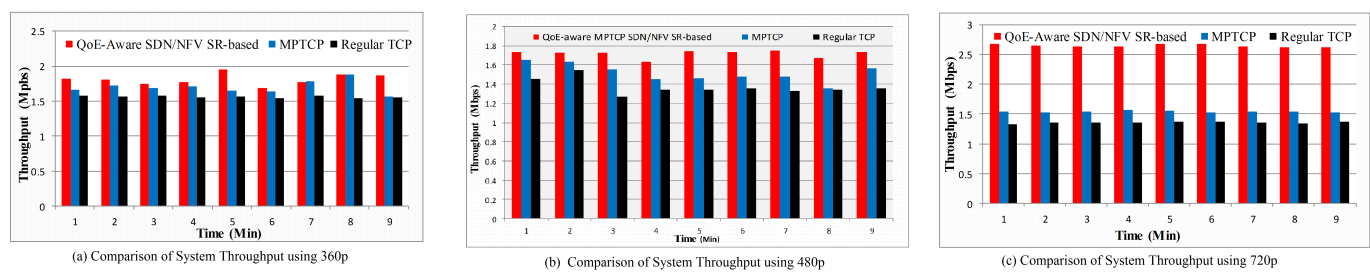

Fig. 3. Comparison of System Throughput for a video resolution of 360p, 480p and 720p
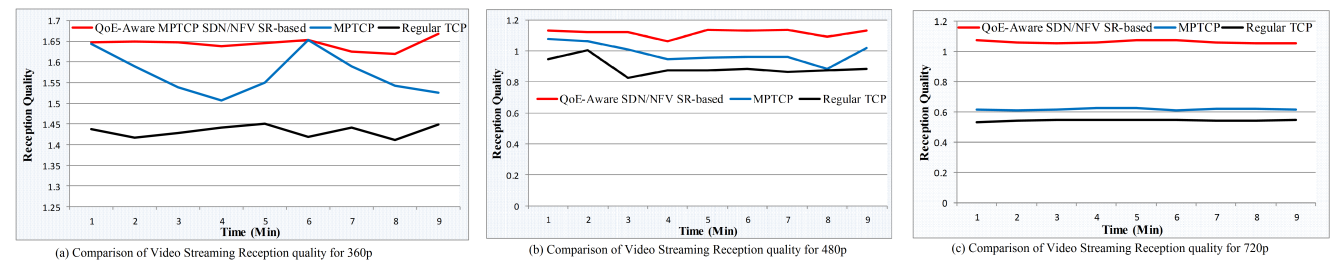

Fig. 4. Comparison of Video Streaming Reception for 360p, 480p and 720p

TABLE II

AVERAGE THROUGHPUT IN MBPS

\begin{tabular}{|l|l|l|l|}
\hline Video Resolution & Our proposal & MPTCP & TCP \\
\hline $360 \mathrm{p}$ & 1.87 & 1.73 & 1.55 \\
\hline $480 \mathrm{p}$ & 1.76 & 1.61 & 1.36 \\
\hline $720 \mathrm{p}$ & 2.66 & 1.56 & 1.37 \\
\hline
\end{tabular}

streaming reception quality using resolution of 360p, 480p and 720 p. It is clear that, the QoE-aware MPTCP/SR SDN/NFVbased approach performs better compared to MPTCP and regular TCP for all three used video resolutions.

\section{CONCLUSION}

In this paper, we first propose a QoE-aware MPTCP SDN/NFV SR-based system that achieve an optimized E2E QoE-level for the end-users. To improve the video quality, we propose to use multiple shortest paths for MPTCP subflows transmission and choose important intermediate nodes to perform source routing using SR paradigm. The aim is to meet future networks bandwidth aggregation and provide an efficient orchestration, QoE control and management of future multimedia services in future networks (e.g., 5G). Preliminary results shows that, our approach outperforms the MPTCP and regular TCP in terms of system throughput and the end-user's QoE.

\section{ACKNOWLEDGMENT}

The work presented in this paper is fully funded by the European Union in the context of Horizon2020 Research and Innovation Programme under Marie Sklodowska-Curie Innovative Training Networks (MSCA-ITN-2014-ETN), Grant Agreement No.643072, Network QoE-NET.

\section{REFERENCES}

[1] J. Wu, C. Yuen, B. Cheng, M. Wang, and J. Chen, "Streaming HighQuality Mobile Video with Multipath TCP in Heterogeneous Wireless Networks," IEEE Transactions on Mobile Computing, vol. 15, no. 9, pp. 2345-2361, March 2016.
[2] A. A. Barakabitze, L. Sun, I.-H. Mkwawa, and E. Ifeachor, "A Novel QoE-Centric SDN-based Multipath Routing Approach of Mutimedia Services over 5G Networks „" IEEE International Conference on Communications, May 2018.

[3] "MPTCP Linux Kernel Implementation," [Available at]:http://www.multipath-tcp.org/.

[4] Y. Liu, Y. Li, M. Caniniy, Y. Wang, and J. Yuan, "Scheduling multi-flow network updates in Software-Defined NFV systems,", IEEE Conference on Computer Communications Workshops (INFOCOM WKSHPS),, April 2016.

[5] N. Kukreja, G. Maier, R. Alvizu, and A. Pattavina, "SDN based Automated Testbed for Evaluating Multipath TCP,," IEEE International Conference on Communications Workshops (ICC),, pp. 718-723, May 2016.

[6] L. Luo, H. Yu, and S. Luo, "Achieving Fast and Lightweight SDN Updates with Segment Routing,," IEEE Global Communications Conference (GLOBECOM), pp. 1-6, December 2016.

[7] L. Davoli, L. Veltri, P. L. Ventre, G. Siracusano, and S. Salsano, "Traffic Engineering with Segment Routing:SDN-based Architectural Design and Open Source Implementation," Fourth European Workshop on Software Defined Networks (EWSDN),, September 2015.

[8] K. D. Joshi and K. Kataoka, "SFO: SubFlow Optimizer for MPTCP in SDN,", 26th IEEE International Telecommunication Networks and Applications Conference (ITNAC) ,, pp. 173-178, December 2016.

[9] S. Chung, S. Moon, and S. Kim, "The virtualized MPTCP proxy performance in cellular network ," Ninth International Conference on Ubiquitous and Future Networks (ICUFN), ,, pp. 703-707, July 2017.

[10] C. Thaenchaikun and W. Panichpattanakul, "Control-plane OpenFlow segment routing SDN for network control messages reduction,", 14th IEEE International Conference on Electrical Engineering/Electronics, Computer, Telecommunications and Information Technology (ECTICON) ,, pp. 322-325, June 2017.

[11] G. Trimponias, Y. Xiao, H. Xu, X. Wu, and Y. Geng, "On Traffic Engineering with Segment Routing in SDN based WANs," IEEE/ACM Trans. Networking, vol. 25, no. 5, pp. 1-17, September 2017.

[12] F. Ongaro, E. Cerqueira, L. Foschini, A. Corradi, and M. Gerla, "Enhancing the Quality Level Support for Real-time Multimedia Applications in Software-Defined Networks ,", International Conference on Computing, Networking and Communications, Communication QoS and System Modeling Symposium, pp. 505-509, June 2015.

[13] P. L. Ventre, "SDN-TE-SR-tools,", https://github.com/netgroup/SDNTE-SR, September 2015.

[14] ITEC, "Dynamic Adaptive Streaming over HTTP:Dash plugin for vlc,", [Available at]:http://www-itec.uni-klu.ac.at/dash/?page_id=10, June 2012.

[15] T. Hofeld, R. Schatz, E. Biersack, and L. Plissonneau, "Internet video delivery in YouTube: from traffic measurements to quality of experience. In: Biersack, E., Callegari, C., Matijasevic, M. (Eds.), Data Traffic Monitoring and Analysis. Springer „” pp. 264-301, June 2013. 